\title{
Modelo para la estimación de potencia eléctrica en módulos fotovoltaicos de tecnología basada en silicio
}

\author{
Model for electric power estimation in photovoltaic silicon modules \\ Francisco Javier Eraso $^{1 *} \quad$ Olger Ferledy Erazo ${ }^{1} \quad$ Edisson Escobar $^{1}$ \\ Recibido 25 de mayo de 2017, aceptado 24 de mayo de 2018 \\ Received: May 25, 2017 Accepted: May 24, 2018
}

\begin{abstract}
RESUMEN
Los módulos fotovoltaicos presentan una capacidad de generación de potencia dependiente de las condiciones climatológicas de su sitio de ubicación. Este trabajo desarrolla y evalúa un modelo que permite determinar la potencia que generaran los módulos solares basados en tecnologías de silicio, monocristalino, policristalino y amorfo de película delgada, si se conocen las condiciones de temperatura e irradiancia de la zona en la cual planean ser ubicados. El modelo se realizó analizando la respuesta en potencia de diferentes módulos solares ante el comportamiento climatológico de una zona durante un año (datos recolectados por un sistema meteorológico). Los parámetros del modelo se calcularon utilizando el algoritmo cuasi Newton con actualización BFGS. La respuesta en potencia presentada por el modelo se comparó con la respuesta generada por un sistema fotovoltaico real que incluye los tres tipos de tecnología alimentando una carga D.C. evidenciando que la respuesta real corresponde a la estimación entregada por el modelo.
\end{abstract}

Palabras clave: Algoritmo cuasi Newton con actualización BFGS, estimación de potencia, modelo FV, módulos FV-Si.

\begin{abstract}
The photovoltaic modules have a power generation capacity dependent on the location site's weather conditions. This work develops and evaluates a model that determines the power generated by solar modules based on monocrystalline, polycrystalline and an amorphous thin silicon film, if the temperature and irradiance conditions of the zone in which they are planned to be located are known. The model was carried out by analyzing the solar modules potential response to the climatological behavior, during a year, Measures collected by a meteorological system; the model parameters were calculated applying the quasi-Newton with BFGS update algorithm. The power model response was compared to the real photovoltaic generation system that includes the three technologies and a D.C. load, evidencing that the real response corresponds to the estimation delivered by the model.
\end{abstract}

Keywords: Quasi Newton BFGS algorithm, PV model, power estimation, Si-PV model.

\section{INTRODUCCIÓN}

La necesidad de suplir una demanda energética cada vez mayor, motiva a las naciones a modificar su matriz energética [1], vinculando fuentes renovables que contribuyen a la disminución de la emisión de gases contaminantes causantes del efecto invernadero [2].

\footnotetext{
1 Facultad de Ingeniería. Institución Universitaria CESMAG. Carrera 20a 14-54. San Juan de Pasto, Colombia. E-mail: oferazo@iucesmag.edu.co; eescobar@iucesmag.edu.co; feraso@iucesmag.edu.co

* Autor de correspondencia
} 
$\mathrm{Al}$ aparecer las fuentes renovables en la canasta de generación, se hace necesario desarrollar estudios tendientes a determinar cuál de ellas se acopla de manera adecuada a cada región [3]. Por practicidad en la instalación, costos de adquisición y modularidad, la energía fotovoltaica se ha convertido en una solución atractiva para cualquier región a nivel mundial; aún más en latitudes cercanas al Ecuador, en donde los niveles de radiación son relativamente constantes durante todo el año.

La generación energética fotovoltaica se realiza aprovechando el efecto fotoeléctrico, a través de diferentes tecnologías como: materiales semiconductores de oblea, semiconductores de lámina delgada, materiales orgánicos o puntos de quantum. Cada tecnología presenta diferencias en la curva de potencia obtenida bajo unas mismas condiciones climatológicas. El comportamiento de estas tecnologías puede ser analizado a través del uso de un modelo matemático; de esta forma, en una determinada región, se implementaría la tecnología que mejor desempeño presente.

Este trabajo modela matemáticamente el comportamiento eléctrico de las tecnologías fotovoltaicas basadas en silicio (monocristalino, policristalino y amorfo de película delgada) utilizando las ecuaciones planteadas en el modelo de Gow [4], éstas se alimentan de los datos de temperatura, irradiancia, voltaje y corriente medidos en una zona determinada durante un periodo de un año y se procesan mediante la aplicación del algoritmo cuasi Newton con actualización BFGS [5-6].

La respuesta que presenta cada modelo, se ajusta al comportamiento real de las tres tecnologías fotovoltaicas que fueron analizadas.

\section{ELEMENTOS TEÓRICOS}

\section{Tecnologías de generación fotovoltaica basadas en silicio}

La tecnología de celdas fotovoltaicas dominante en el mercado se basa en silicio, elemento que sirve de base para la mayoría de componentes electrónicos y que se encuentra en forma de una roca de óxido de silicio llamada cuarcita [7]. Las celdas de silicio se pueden fabricar por medio de obleas monocristalinas, policristalinas o películas delgadas amorfas o nanocristalinas.

\section{Tecnología de silicio monocristalino}

Las celdas monocristalinas, se forman a partir un sólido de silicio cristalino intacto, sin bordes granulados y con una estructura cristalina cúbica de diamante en la cual, los átomos se encuentran organizados siguiendo un patrón repetitivo y simétrico, aunque no en la misma dirección [7].

Para construir una celda solar se crean obleas tipo $\mathrm{N}$ o tipo $\mathrm{P}$. De acuerdo a Lin, la región tipo $\mathrm{N}$ se llama emisor y se encuentra altamente dopada, sobre esta región se ubican unos contactos metálicos y una capa antirreflejo generalmente fabricada de silicio nitridado, que permite incrementar la transmitancia de la luz. Cuando la luz impacta la celda, la energía de un fotón genera un par electrón hueco en la región tipo P [8], estos portadores de carga se dirigen a los contactos metálicos permitiendo el flujo de corriente.

\section{Tecnología de silicio policristalino}

"Debido a que la industria fotovoltaica no requiere que el silicio tenga la misma pureza de los componentes de la microelectrónica, se hace posible utilizar el silicio sobrante de la producción en la fabricación de celdas fotovoltaicas" [9]. Las celdas policristalinas se construyen a base de pequeños granos cristalinos que provienen de diferentes fuentes en donde sus átomos se encuentran organizados con orientaciones aleatorias, estos se funden en un crisol y se solidifican. La calidad de este tipo de celdas no es tan buena como la del silicio monocristalino, porque presenta gran cantidad de defectos en sus bordes; sin embargo, los costos de fabricación son mucho menores. El hecho de formar celdas debido a la solidificación de crisoles de gran tamaño también permite que el costo de producción sea más económico.

\section{Tecnología de silicio amorfo}

Esta tecnología se desarrolla sobre una película delgada de silicio amorfo como material absorbente con substratos de vidrio u otro material flexible [7-9], y no sobre obleas como es el caso del silicio monocristalino y policristalino. Una diferencia importante entre estos dos tipos de materiales consiste en que los materiales de película delgada poseen una banda prohibida directa, es decir que hay alineación directa entre el tope inferior de la banda de conducción y el superior de la banda de valencia [7]. 
A diferencia del silicio cristalino que tiene una estructura atómica ordenada, el silicio amorfo posee una estructura tetraédrica desordenada en una red aleatoria, en donde la organización de los átomos no luce como un cristal [7].

De acuerdo a Lin: la estructura básica de estos dispositivos es P-i-N (una capa tipo P, una capa intrínseca y una capa tipo $N$ ). En la capa tipo $P$ ocurre un proceso de degradación de la luz denominado efecto Staebler-Wronski, razón por la cual se necesita la capa intrínseca con diferentes bandas prohibidas [8]. En esta estructura la absorción de fotones ocurre en la capa intrínseca, lo que permite incrementar el nivel de absorción de fotones y el tiempo de vida de los portadores de carga.

\section{Factores climáticos incidentes en la generación fotovoltaica}

Teniendo en cuenta que el planeta cuenta con dos movimientos, uno alrededor de su eje, denominado rotación y otro llamado traslación, el cual se efectúa alrededor del Sol describiendo una elipse con $23^{\circ} 27^{\prime}$ de inclinación, se observa que la luz proveniente del Sol no es uniforme en las diferentes zonas del Tierra lo que ocasiona la variación de los factores climáticos y por lo tanto la forma en la cual los seres vivos perciben el clima en cada una de las regiones del planeta [10].

Los factores que tienen incidencia directa en la generación de energía son la temperatura y la irradiancia, a partir de estos se alimenta el modelo de la celda fotovoltaica.

\section{Temperatura}

La temperatura es una magnitud relacionada con la rapidez del movimiento de las partículas que constituyen la materia, a mayor agitación mayor temperatura. Es importante tener en cuenta que no se debe confundir la temperatura con el calor, siendo este último una forma de energía "producida por la vibración acelerada de las moléculas que componen un cuerpo" [11].

De acuerdo a Escudero, de la energía que llega a la Tierra proveniente del Sol en forma de radiación solar de onda corta solo el $45 \%$ es absorbido y transformado en forma de calor, el resto es reflejado al espacio. La Tierra es un cuerpo que irradia energía en onda larga de tal forma que el calor ganado de la radiación incidente sea igual al perdido en la radiación terrestre para evitar sobrecalentamiento y mantener un balance térmico [12].

La capacidad calorífica del suelo permite absorber o emitir radiación con mayor o menor dificultad, en este sentido la temperatura en el agua, el desierto o la selva va a diferir a pesar que la radiación percibida sea la misma. Para este trabajo la temperatura se mide en grados Celsius $\left({ }^{\circ} \mathrm{C}\right)$.

\section{Irradiancia}

La radiación electromagnética que emana del Sol se convierte en la principal fuente de energía en la Tierra, "prácticamente toda la energía que llega a la atmósfera y a la Tierra procede del exterior emitida por el Sol. Rige y gobierna todos los fenómenos de la naturaleza, el clima la circulación general de la atmósfera, los movimientos oceánicos excluyendo las mareas, el ciclo hidrológico y los combustibles como el petróleo y el carbón" [11]. Esta energía se llama irradiancia $(G)$ y se define como la potencia por unidad de área normal a la dirección de propagación expresada en vatios por metro cuadrado [13]; se encuentra en una longitud de onda comprendida entre $150 \mathrm{~nm}$ y $4000 \mathrm{~nm}$, un $9 \%$ corresponde a radiación ultravioleta, $45 \%$ radiación visible y $46 \%$ radiación infrarroja [14-15].

\section{Modelo de una celda fotovoltaica}

Una celda fotovoltaica basada en silicio, permite convertir la energía proveniente del Sol en energía eléctrica. Esta energía incide en la celda solar generando un par electrón hueco que se colecta en las terminales metálicas de la celda y permite el flujo de la corriente eléctrica [9].

El modelo de la celda está compuesto por una fuente de corriente $\left(\mathrm{I}_{\mathrm{PH}}\right)$ que representa la corriente foto generada, es decir la corriente que se produce por incidencia de la radiación solar; también incluye un diodo resultante de la unión PN y un par de resistencias parásitas, la primera llamada $R_{s}$ (resistencia serie) que se obtiene de la oposición al paso de la corriente propia del material conductor de los contactos metálicos, la unión del semiconductor PN y la interfaz entre el semiconductor y los contactos metálicos; la segunda llamada $\mathrm{R}_{\mathrm{sh}}$ (resistencia shunt o paralelo), que se debe a defectos macroscópicos en la celda solar que provee un camino alternativo para la generación de fotocorriente [16]. El circuito 
equivalente del modelo de la celda solar se representa en la Figura 1.

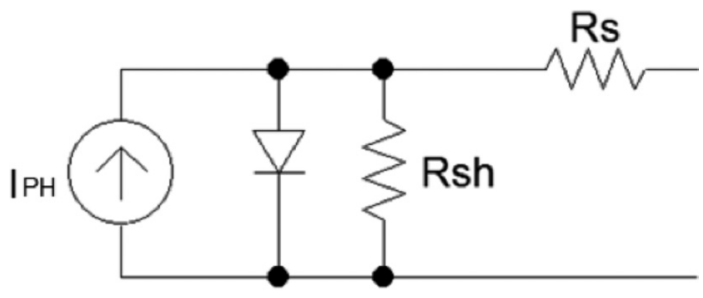

Figura 1. Circuito equivalente de una celda solar.

Según Lien, la corriente foto generada se debe a la generación del par hueco electrón en un volumen [16], por lo tanto la ecuación (1) determina está corriente en función del volumen.

$$
I_{P H}=Q A G_{0}\left(L_{P}+L_{N}\right)
$$

En donde:

q: es la carga elemental cuyo valor es constante e igual a $1,6 \times 10^{-19} \mathrm{C}$.

A: Es el área transeccional del diodo o la celda solar.

$\mathrm{G}_{\mathrm{o}}$ : Es la tasa de generación o concentración de fotones, es decir el número de fotones inyectados por segundo al material semiconductor.

$\mathrm{L}_{\mathrm{p}}$ : Es la longitud de difusión para los huecos

$\mathrm{L}_{\mathrm{n}}$ : Es la longitud de difusión para los electrones

Debido a que la generación de corriente en la celda depende del área de la misma, es muy común utilizar el término densidad de corriente (J) en lugar de la corriente (I). Este término hace referencia a la corriente generada por unidad de área y se expresa en términos de $\mathrm{mA} / \mathrm{cm}^{2}$. La ecuación (2) plantea el modelo real de la celda fotovoltaica, teniendo en cuenta la unión PN y las resistencias parásitas.

$$
J=N_{P}\left[-J_{P H}+I_{S}\left(e \frac{q\left(\frac{V_{A}}{N_{S}}-J R_{S}\right)}{N_{S}}-1\right)+\left(\frac{\frac{V_{A}}{N_{S}}}{R_{S H}}-J R_{S}\right)\right]
$$

Los modelos matemáticos que estiman el comportamiento eléctrico de las tecnologías monocristalina y policristalina pueden utilizar el modelo de doble exponencial, expresado en la ecuación (3), que de acuerdo a Luque [17] corresponde a una representación que determina la corriente a través del módulo fotovoltaico teniendo en cuenta los parámetros de resistencia paralelo $\left(\mathrm{R}_{\mathrm{sh}}\right)$ y resistencia serie $\left(\mathrm{R}_{\mathrm{s}}\right)$ de todo el conjunto de celdas fotovoltaicas que componen un módulo. De acuerdo a Gow, este modelo representa de manera aceptable el comportamiento de módulos solares, especialmente de tecnología policristalina, sin embargo, para celdas construidas a base de silicio amorfo que no presentan un cambio pronunciado en la relación voltaje corriente: el efecto de la corriente de saturación $\mathrm{I}_{\mathrm{S} 1}$ es equivalente a cero [17] y por lo tanto se utiliza la ecuación (2).

$$
J=N_{P}\left[\begin{array}{c}
-J_{P H}+J_{S 1}\left(e \frac{q\left(\frac{V_{A}}{N_{S}}-J R_{S}\right)}{K T}-1\right)+ \\
J_{S 2}\left(e \frac{q\left(\frac{V_{A}}{N_{S}}-J R_{S}\right)}{A K T}-1\right)+\frac{\left(\frac{V_{A}}{N_{S}}-J R_{S}\right)}{R_{S H}}
\end{array}\right]
$$

Tanto para las ecuaciones (2) y (3):

J corresponde a la densidad corriente entregada por el módulo solar.

$\mathrm{J}_{\mathrm{PH}}$ : es la densidad de corriente fotogenerada.

$\mathrm{J}_{\mathrm{S} 1}$ : densidad de corriente generada debido al mecanismo de difusión.

$\mathrm{J}_{\mathrm{S} 2}$ : densidad de corriente generada debido al proceso de recombinación de portadores minoritarios de carga.

q: es la carga elemental cuyo valor es constante e igual a $1,6 \times 10^{-19} \mathrm{C}$.

$\mathrm{V}_{\mathrm{A}}$ : Es el voltaje en el módulo.

K: Es la constate de Boltzmann igual a 1,38 $\times 10^{-23}$ $\mathrm{J} / \mathrm{K}$.

T: es la temperatura ambiente cercana a $25^{\circ} \mathrm{C}$.

$\mathrm{N}_{\mathrm{S}}$ : Contiene la cantidad de celdas fotovoltaicas del módulo conectadas en serie.

$\mathrm{N}_{\mathrm{P}}$ : Contiene la cantidad de celdas fotovoltaicas del módulo conectadas en paralelo.

$\mathrm{R}_{\mathrm{S}}$ : corresponde a la resistencia serie asociada al módulo fotovoltaico.

$\mathrm{R}_{\mathrm{SH}}$ : hace referencia a la resistencia paralelo o shunt del módulo fotovoltaico. 
A: contiene el valor del factor de idealidad o calidad del diodo.

A partir de los resultados de corriente y voltaje obtenidos del modelo se calcula la potencia la densidad de potencia que entrega cada tecnología, siendo esta última la potencia generada por unidad de área para cada tecnología [18].

\section{Parámetros de evaluación de celdas solares}

Si se requiere comparar el desempeño de diferentes tecnologías, lo primero que se debe tener en cuenta es que las condiciones a las que se encuentren sometidas sean las mismas [13]. En este sentido todas las características de un módulo solar entregadas por un fabricante provienen de pruebas realizada bajo unos estándares de prueba conocido como STC (Standard Test Conditions).

Usualmente los fabricantes entregan las curvas de densidad de corriente contra voltaje (J-V), las cuales se toman siguiendo las siguientes condiciones: condición de temperatura igual a $25^{\circ}$ Celsius; condición de potencia de entrada, representada por la irradiancia, igual a $1000 \mathrm{~W} / \mathrm{m}^{2}$; condición del espectro solar que llega a la superficie de la tierra, representado por una masa de aire igual a 1,5 AM, equivalentes a una latitud de $48,2^{\circ}$ (ángulo con respecto a la horizontal).

\section{Eficiencia}

La eficiencia de conversión ( $\eta$ ), es la relación existente entre la energía generada por la celda solar con respecto a la energía incidente (irradiancia). El cálculo de la eficiencia se realiza mediante la ecuación (4), en la cual se observa como la potencia de salida $\left(\mathrm{P}_{\text {out }}\right)$ es el producto de la densidad de corriente máxima $\left(\mathrm{J}_{\mathrm{mp}}\right)$ y el voltaje máximo $\left(\mathrm{V}_{\mathrm{mp}}\right)$ en la celda solar [7-8].

$$
\eta=\frac{P_{\text {out }}}{P_{\text {in }}}=\frac{P_{\max }}{P_{\text {in }}}=\frac{J_{m p} V_{m p}}{P_{\text {in }}}
$$

\section{METODOLOGÍA}

\section{Recolección de datos}

El proceso de recolección de datos se realizó a través de dos etapas paralelas, la primera consiste en recibir la información meteorológica registrada por la estación meteorológica DAVIS Advantage PRO 2 a través del programa Weather Link. Los instrumentos de medición cumplen con los requisitos de la organización Meteorológica Mundial [19]. Esta información se entrega a la base de datos denominada RAMPA FOTOVOLTAICA; la segunda etapa registra el valor de voltaje y corriente proveniente de los módulos solares conectados a cargas resistivas, estos datos también se envían a la base de datos para efectuar un análisis con herramientas estadísticas.

La información se recolectó durante un periodo de un año, para una localización que cuenta con irradiancia promedio igual a $391,7 \mathrm{~W} / \mathrm{m}^{2}$ y una temperatura promedio de $16,8^{\circ} \mathrm{C}$.

\section{Procesamiento de la información}

El modelo que representa el comportamiento de los módulos fotovoltaicos determinado por las ecuaciones (2) y (3), tiene características no lineales y se resuelve de manera heurística mediante la aplicación del algoritmo cuasi Newton con actualización BFGS, programado en Matlab® que se alimenta con todos los datos recolectados.

\section{Comparación densidad de potencia}

Los valores de densidad de potencia obtenidos del modelo, se contrastan con los reales para los valores promedio de irradiancia y temperatura en la zona. Finalmente, se calcula el error cuadrático medio para determinar la confiabilidad del modelo.

\section{RESULTADOS}

El modelo se obtiene de la respuesta de voltaje y corriente de los módulos fotovoltaicos, encontrada para los valores de irradiancia y temperatura recolectados. A partir de ellos, se calcula las corrientes $\mathrm{I}_{\mathrm{PH}}, \mathrm{I}_{\mathrm{S} 1}, \mathrm{I}_{\mathrm{S} 2}$, las resistencias paralelo $\mathrm{R}_{\mathrm{SH}} \mathrm{y}$ serie $\mathrm{R}_{\mathrm{s}}$ y el factor de idealidad A en las ecuaciones (2) y (3) las cuales son no lineales y no pueden ser resueltas de manera analítica, por esta razón se utilizó un método heurístico denominado algoritmo cuasi Newton con actualización BFGS que difiere del algoritmo Newton Raphson utilizado por Gow [4-6].

Los parámetros dependientes ( $\mathrm{I}_{\mathrm{PH}}, \mathrm{I}_{\mathrm{S} 1}, \mathrm{I}_{\mathrm{S} 2}, \mathrm{R}_{\mathrm{SH}}, \mathrm{R}_{\mathrm{s}}$ y A) se obtienen aplicando las ecuaciones 5 a 10 planteadas por Gow. Estos parámetros se encuentran en función de la irradiancia $(\mathrm{G})$, la temperatura (T) y unas constantes $\mathrm{K}_{0}$ a $\mathrm{K}_{12}$ que se obtienen minimizando el error cuadrático entre los valores estimados en el modelo y los valores de corriente 
y voltaje recopilados. Los resultados se presentan en la Tabla 1.

$$
\begin{gathered}
I_{P H}=k_{0} G\left(1+k_{1} T\right) \\
I_{S 1}=k_{2} T^{3} T e \frac{k_{3}}{T} \\
S_{S 2}=k_{4} T \frac{3}{2} T e \frac{k_{5}}{T} \\
A=k_{6}+k_{7} T \\
R_{S}=\left(k_{8}+\frac{k_{9}}{G}+k_{10} T\right) \\
R_{S H}=k_{11} e^{k_{12} T}
\end{gathered}
$$

Los parámetros dependientes resultantes para una irradiancia igual a $391,7 \mathrm{~W} / \mathrm{m}^{2}$ y una temperatura igual a $16,8^{\circ} \mathrm{C}$ se presentan en la Tabla 2. Estos valores corresponden al promedio de irradiancia y temperatura registrados en la zona de estudio [13].

\section{DENSIDAD DE POTENCIA}

La comparación de las gráficas obtenidas durante un año de evaluación se realiza utilizando los valores proyectados por el modelo y su contraste con el comportamiento real.

Se evidencia que la tecnología monocristalina presenta un mejor comportamiento que las otras tecnologías y que la tecnología de silicio amorfo a pesar de ser la que menor potencia por unidad de área entrega, presenta una mayor estabilidad que las demás.

Tabla 1. Parámetros A y k para las tecnologías de módulos solares basadas en silicio de la zona de estudio.

\begin{tabular}{|c|c|c|c|}
\hline \multirow{2}{*}{ Parámetro } & \multicolumn{3}{|c|}{ Tecnología } \\
\cline { 2 - 4 } & Monocristalina & Policristalina & Si Amorfo \\
\hline $\mathrm{A}$ & 2 & 2 & 2 \\
\hline $\mathrm{K}_{0}$ & $-6,62 \times 10^{-5}$ & $-7,68 \times 10^{-5}$ & $-8,31 \times 10^{-5}$ \\
\hline $\mathrm{K}_{1}$ & $-0,1098$ & $-0,1098$ & - \\
\hline $\mathrm{K}_{2}$ & 44,5355 & 44,5355 & - \\
\hline $\mathrm{K}_{3}$ & -12640 & -12640 & -12640 \\
\hline $\mathrm{K}_{4}$ & 11,8003 & 11,8003 & 11,8003 \\
\hline $\mathrm{K}_{5}$ & $-7317,4$ & $-7317,4$ & $-7317,4$ \\
\hline $\mathrm{K}_{6}$ & 2 & 2 & 2 \\
\hline $\mathrm{K}_{7}$ & 0,0028 & $1,703 \times 10^{-8}$ & $1,13 \times 10^{-8}$ \\
\hline $\mathrm{K}_{8}$ & 0,0132 & 0,0147 & 0,0147 \\
\hline $\mathrm{K}_{9}$ & 16,126 & 16,126 & 16,126 \\
\hline $\mathrm{K}_{10}$ & $-0,4253$ & $-4,53 \times 10^{-5}$ & $-4,48 \times 10^{-5}$ \\
\hline $\mathrm{K}_{11}$ & 2303000 & 2303000 & 2303000 \\
\hline $\mathrm{K}_{12}$ & $-0,0281$ & $-0,02812$ & $-0,02812$ \\
\hline
\end{tabular}

Tabla 2. Parámetros de las tecnologías fotovoltaicas evaluadas para las condiciones de $\mathrm{G}=391,7 \mathrm{~W} / \mathrm{m}^{2}$ y $\mathrm{T}=16,8^{\circ} \mathrm{C}$.

\begin{tabular}{|l|c|c|c|}
\hline \multirow{2}{*}{ Parámetro } & \multicolumn{3}{|c|}{ Tecnología } \\
\cline { 2 - 4 } & Monocristalina & Policristalina & Si Amorfo \\
\hline $\mathrm{I}_{\mathrm{PH}}(\mathrm{A})$ & 0,9784 & 0,9274 & 1,0031 \\
\hline $\mathrm{I}_{\mathrm{S} 1}(\mathrm{~A})$ & $1,237 \times 10^{-10}$ & $1,238 \times 10^{-10}$ & - \\
\hline $\mathrm{I}_{\mathrm{S} 2}(\mathrm{~A})$ & $6,297 \times 10^{-7}$ & $6,297 \times 10^{-7}$ & $6,2977 \times 10^{-7}$ \\
\hline $\mathrm{R}_{\mathrm{S}}(\Omega)$ & 0,0559 & 0,0427 & 0,0429 \\
\hline $\mathrm{R}_{\mathrm{SH}}(\Omega)$ & 665,509 & 665,5059 & 665,5058 \\
\hline $\mathrm{P}\left(\mathrm{W} / \mathrm{M}^{2}\right)$ & 30,82 & 27,24 & 15,41 \\
\hline
\end{tabular}


La densidad de potencia varía de acuerdo a las combinaciones de irradiancia y temperatura que inciden en sistema fotovoltaico. La Figura 2, presenta la curva de densidad de corriente contra voltaje obtenida para las condiciones de climatológicas promedio de la zona estudiada (irradiancia promedio igual a $391,7 \mathrm{~W} / \mathrm{m}^{2}$ y una temperatura promedio de $\left.16,8^{\circ} \mathrm{C}\right)$.

El punto de máxima transferencia de potencia se encuentra en la denominada rodilla de la curva, para llegar a este punto se puede desarrollar un seguidor de Máxima Transferencia de Potencia.

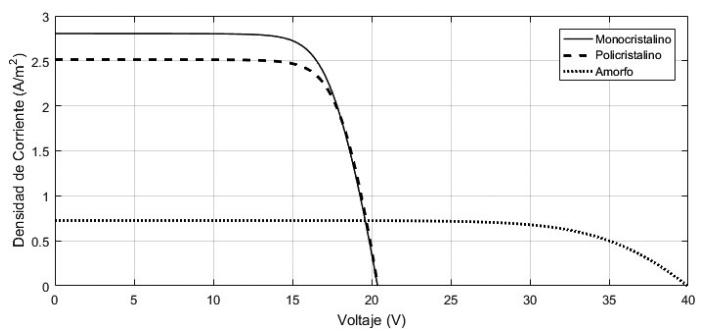

Figura 2. Relación corriente y voltaje para $\mathrm{G}=$ $391,7 \mathrm{~W} / \mathrm{m}^{2}$ y $\mathrm{T}=16,8^{\circ} \mathrm{C}$ en la zona de estudio.

Las tecnologías monocristalina y policristalina utilizadas tienen un valor de Voltaje de Circuito Abierto $\left(\mathrm{V}_{\mathrm{OC}}\right)$ igual a 20 voltios y la tecnología de silicio amorfo, de 40 voltios.

A partir de la curva de densidad corriente y voltaje, se calcula la curva de densidad de potencia para cada una de las tecnologías evaluadas, siendo las tecnologías monocristalina y policristalina las de mejor respuesta, como se observa en la Figura 3.

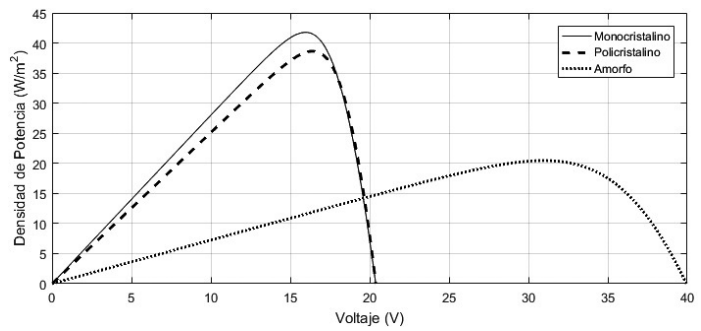

Figura 3. Relación potencia y voltaje para $\mathrm{G}=$ $391,7 \mathrm{~W} / \mathrm{m}^{2}$ y $\mathrm{T}=16,8^{\circ} \mathrm{C}$.

\section{EVALUACIÓN DEL MODELO}

\section{Respuesta en potencia}

El modelo se alimenta con valores reales de irradiancia y temperatura, medidos con la estación meteorológica y se compara la respuesta de potencia generada por el modelo con la respuesta real entregada por cada tecnología fotovoltaica.

La Figura 4 presenta los valores reales y simulados de voltaje y corriente de la tecnología monocristalina, en esta se puede observar como el modelo realiza un pronóstico adecuado del comportamiento del módulo fotovoltaico porque el error cuadrático medio alcanzado es igual a 0,0069 .

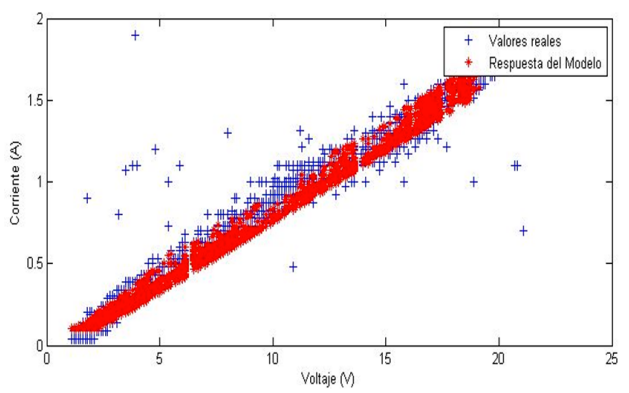

Figura 4. Evaluación del modelo matemático para la tecnología de silicio monocristalino.

De igual manera, la predicción de corriente y voltaje que realiza el modelo desarrollado para la tecnología de silicio policristalino (Figura 5) es similar a la realidad, con un error cuadrático medio igual a 0,0685 .

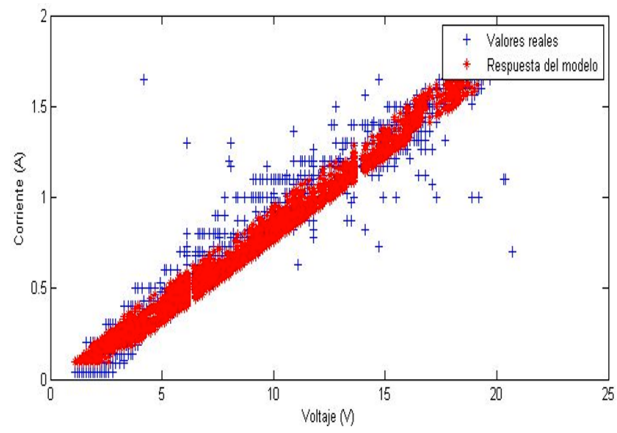

Figura 5. Evaluación del modelo matemático para la tecnología de silico policristralino.

Finalmente, la Figura 6 evidencia el comportamiento del modelo y la respuesta real de la tecnología de silicio amorfo, los resultados obtenidos validan el 


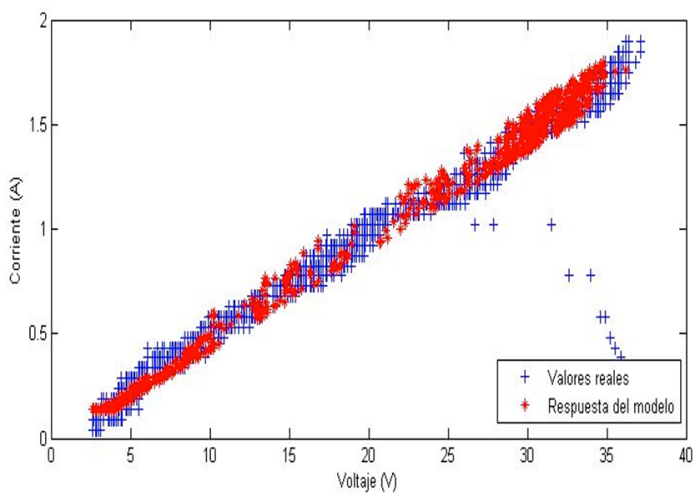

Figura 6. Evaluación del modelo matemático para la tecnología de silico amorfo.

modelo al encontrarse un error cuadrático medio alcanzado igual a 0,1317 .

Dado el error cuadrático medio encontrado en la evaluación de los tres tipos de tecnologías (Figuras 4 a 6), se puede deducir que la potencia generada por el modelo es acorde a la entregada por los módulos instalados de manera real. Esto se comprueba en la Figura 7, en donde se comparó la respuesta del modelo desarrollado para la tecnología monocristalina (línea azul), la tecnología más popular, con las mediciones recopiladas de la instalación fotovoltaica (línea verde), obteniendo un coeficiente de correlación de 0,9824 y uno de determinación igual a 0,965. Esto indica que el comportamiento de la potencia en los dos casos tiene una correspondencia muy alta.

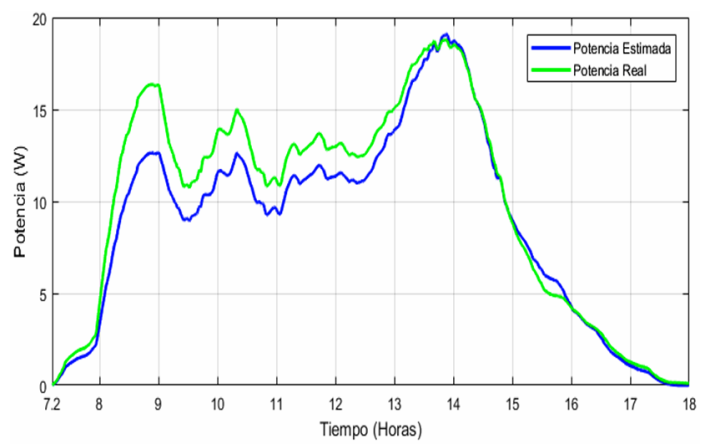

Figura 7. Respuesta en potencia tecnología monocristalina (valor estimado y real) para el 8 de julio de 2015.

La Figura 7, presenta un ejemplo de lo anterior para el día 8 de julio de 2015. En este día la predicción de energía total generada por esta tecnología era de $45 \mathrm{kWh}$ y en realidad la energía generada fue de $50 \mathrm{kWh}$.

\section{Eficiencia}

La Figura 8 resume la eficiencia promedio en la generación de energía para cada una de las tecnologías evaluadas de acuerdo a las condiciones meteorológicas de la zona durante el año de evaluación. En esta se observa que la tecnología monocristalina presenta la mayor eficiencia con un $10,8 \%$, seguida de la policristalina con un $10,07 \%$ y finalmente la tecnología de silicio amorfo presenta un 5,74\%.

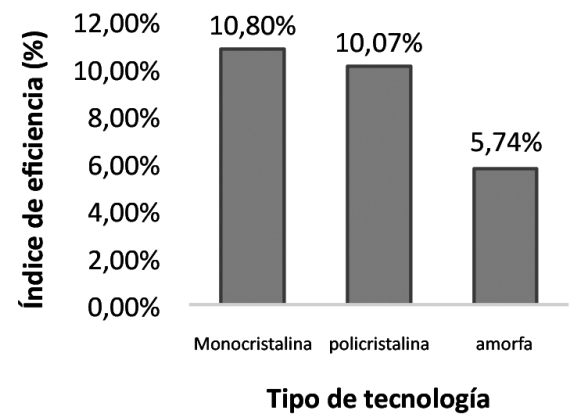

Figura 8. Eficiencia de las tecnologías fotovoltaicas para el año 2014.

\section{CONCLUSIONES}

Las ilustraciones 4, 5 y 6 evidencian la acertada respuesta del modelo fotovoltaico para cada una de las tecnologías evaluadas (silicio monocristalino, policristalino y amorfo de película delgada). La predicción generada por el modelo puede ser considerada como el comportamiento real de cada tecnología ante una entrada de parámetros de irradiancia y temperatura determinados por las condiciones climatológicas de una zona. Si se cuenta con datos de una estación meteorológica, la predicción que genera el modelo permite realizar diseños acertados que permitan suplir la demanda de energía de la ubicación en la que se pretenda realizar una implementación fotovoltaica.

El cálculo de eficiencia es válido para las condiciones climatológicas de la zona de estudio. Si se quiere determinar estos valores en otra zona potencial, se pueden aplicar las ecuaciones con los parámetros de la zona de evaluación. 


\section{AGRADECIMIENTOS}

Los autores expresan su agradecimiento a la Institución Universitaria CESMAG, por el apoyo brindado en la financiación del presente proyecto.

\section{REFERENCIAS}

[1] J. Castro. "Perspectivas de la demanda energética global". Petrotencia. Vol. $1 \mathrm{~N}^{\mathrm{o}} 1$, pp. 54-70. 2011.

[2] J.L. Arvizu. "Registro histórico de los principales países emisores". De Cambio cilmático: una misión desde México, México, Instituto nacional de Ecología. pp. 99. 2004.

[3] F.J.E. Checa, E.E. Rosero and O.F.E. De la Cruz. "Comparison between the energy generated from three types of c-Si photovoltaic modules and the temperature and irradiance of the city of Pasto, Colombia". Innovative Smart Grid Technologies Latin America (ISGT LATAM) IEEE PES. pp. 757-761. 2015.

[4] J.A. Gow and C.D. Manning. "Development of a photovoltaic array model for use in power-electronics simulation studies". Vol. $146 \mathrm{~N}^{\circ}$ 2. 1999.

[5] J.F. Bonnans, J.C. Gilbert and C. Lemaréchal. "Numerical optimization: theoretical and practical aspects". Springer Science \& Business Media, Berlín: Springer-Verlag. 2013.

[6] S. Wright and J. Nocedal. "Numerical optimization". New York: Springer Science + business media. 1999.

[7] A. Smets, Solar Energy: Fundamental, tecnology and systems, Delft: Delft University of Technology. 2014.

[8] C.F. Lin, W.F. Su, C.I. Wu and I.C. Cheng, Organic. "Inorganic and Hybrid Solar Cells".
New Jersey: IEEE Press Editorial Board. Wiley publications. 2012.

[9] L. Fraas, L. Partain, Solar Cells and Their Applications". Segunda ed., New Jersey: Wiley Publications. 2010.

[10] J. Eslava. "Climatología y diversidad climática de Colombia". Vol. 18 № 71, pp. 509. 1993.

[11] M. Ledesma-Jimeno. "Principios de Meteorología y Climatología". Madrid: Paraninfo. 2011.

[12] J.M. Escudero-López. "Manual de Energía Eólica”. Segunda ed. Madrid-México: Ediciones Mundi Prensa. 2011.

[13] F.J. Eraso-Checa, O.F. Erazo y E. Escobar. "Energía Fotovoltaica: Comparación de tecnologías basadas en silicio, Pasto: Tiempos Nuevos. 2017.

[14] K. Jager, Solar Energy: Fundamental, tecnology and systems, Delft: Delft University of Technology. 2014.

[15] W. Stuart, M. Green, M. Watt and R. Corkish. "Applied Photovoltaics". Segunda ed., London-Whasington: Earthscan. 2007.

[16] S. Lien-Chuang. "Physics of photonic devices". New Jersey: Wiley. 2009.

[17] A. Luque y S. Hegedus. "Handbook of photovoltaic science and engineering". West Sussex: Wiley. 2003.

[18] I. Martínez. "Termodinámica de la atmósfera". 2010. Fecha de consulta: 28 de noviembre de 2014. URL: Available: http://biblioteca. universia.net/html_bura/ficha/params/title/ termodinamica-atmosfera/id/54767154.html.

[19] OMM. "Organización Meteorológica Mundial. Manuales". 2008. Fecha de consulta: 7 de agosto de 2013. URL: http:// www.wmo.int/pages/prog/gcos/documents/ gruanmanuals/CIMO/CIMO_Guide-7th_ Edition-2008.pdf. 